EXTENDED REPORT

\title{
Decreased levels of cGMP in vitreous and subretinal fluid from eyes with retinal detachment
}

\section{E C La Heii, H G T Blaauwgeers, J de Vente, M Markerink, A T A Liem, A G H Kessels, H Steinbusch, F Hendrikse}

Br J Ophthalmol 2003;87:1409-1412

Background: Cyclic guanosine monophosphate (cGMP) is produced in different retinal cells, including photoreceptor cells, wherein cGMP mediates photo-transduction. CGMP is degraded by phosphodiesterases (PDE). The aim was to investigate whether retinal detachment alters intraocular CGMP levels in human eyes.

See end of article for authors' affiliations

Methods: cGMP and PDE were determined in vitreous fluid from 50 eyes with a retinal detachment (group I) and in 20 control samples (group II) of vitreous fluid from eyes without retinal detachment. Group III consisted of subretinal fluid samples from 70 eyes with retinal detachment.

Correspondence to: Dr E C La Heii, Department of Ophthalmology, University Hospital Maastricht, PO Box 5800 P Debyelaan 25, 6202 AZ, Maastricht, Netherlands; eela@soog.azm.nl

Accepted for publication 3 March 2003

Results: cGMP in vitreous fluid from eyes with retinal detachment (6.5 (SD 1.7) nM) was decreased compared to controls (67.1 (10.0) $\mathrm{nM})(\mathrm{p}<0.0001)$. In subretinal fluid, the mean level of cGMP was 2.4 (0.2) nM. No PDE could be detected in any of the intraocular fluid samples of patients nor controls. A decrease in the mean level of CGMP in subretinal fluid of eyes with retinal detachment correlated with a longer duration of detachment $(r=-0.45, p=0.007)$.

Conclusions: Retinal detachment was found to be associated with a decrease in vitreous cGMP concentration. In subretinal fluid, a low cGMP level correlated inversely with the duration of the detachment.
$\mathrm{R}$ etinal detachment separates the photoreceptors from the retinal pigment epithelial (RPE) cell layer and the underlying choriocapillaris and therefore from their source of oxygen and glucose. Even if the retina is surgically reattached, final visual acuity may sometimes be low. ${ }^{12}$ Permanent retinal damage may be caused by hypoxia and/ or ischaemia in the detached retina, leading to necrosis and apoptosis of retinal cells, like photoreceptors, RPE cells, and Müller cells. ${ }^{3-5}$

CGMP mediates the metabolic cascade of photoreceptor signal transduction. cGMP is synthesised by guanylyl cyclases (GCs), which are found in photoreceptor cells, ${ }^{6}$ but also in several other inner retinal neurons. ${ }^{7}$ When photopigments absorb light, a metabolic cascade is triggered, resulting in the degradation of cGMP by the enzyme phosphodiesterase (PDE). CGMP is present in lower concentrations in the central (macular) region of the neural retina than in other areas, which corresponds approximately to the distribution of rod photoreceptor cells. ${ }^{8}$

The role of CGMP and PDE in photoreceptors has recently attracted attention, because sidenafil (Viagra) has a mild inhibitory effect on PDE6 in the photoreceptors, causing a temporary accumulation of cGMP. This is probably responsible for the perception of a bluish haze or increased light sensitivity in some patients using Viagra. ${ }^{9}$

In retinal detachment, the intracellular metabolic cascade of phototransduction may be altered, caused by an energy deprivation of the photoreceptors. Because of processes like apoptosis and necrosis after retinal detachment, the number of various other retinal cells expressing CGMP may also be reduced. Both situations may lead to a decreased level of cGMP in the retina and vitreous cavity of eyes with retinal detachment.

Levels of intraocular cGMP have been measured in experimental retinal detachment ${ }^{10}$ but no studies have been published yet on CGMP levels in human eyes with retinal detachment. The purpose of our study was therefore to analyse cGMP levels in ocular fluid samples obtained during vitreoretinal surgery from eyes with retinal detachment, and to compare them with cGMP levels in control eyes without retinal detachment. In the same intraocular fluid samples PDE was also determined since this is responsible for the degradation of cGMP.

\section{MATERIALS AND METHODS \\ Patients}

Vitreous fluid samples from 50 consecutive patients with a retinal detachment who underwent vitrectomy were obtained (group I). Twenty undiluted vitreous samples from 20 eyes without retinal detachment undergoing vitrectomy served as controls (group II). They included eight eyes with idiopathic macular hole and 12 eyes with idiopathic epiretinal membranes. Subretinal fluid samples from 70 consecutive patients with a rhegmatogenous retinal detachment operated on with a conventional scleral buckling technique (group III) were also collected. Because subretinal fluid can only be found under the pathological condition of a retinal detachment, no controls were possible for group III. All patients were operated in our clinic between February 1999 and March 2000. Patients with uveitis, with a traction retinal detachment as a result of proliferative diabetic retinopathy, or with a history of ocular trauma were excluded from our study. Patients were treated with mydriatic drops (phenylephrine hydrochloride 5\% and tropicamide $0.5 \%$ ) $1-2$ hours before surgery. No other local or systemic treatment for retinal detachment was given before surgery. The study was performed with the agreement of the institutional ethics committee; all patients gave their informed consent before inclusion in the study and after the nature of the study and possible consequences were explained. The study was conducted in accordance with the ethical standards laid down in the 1964 Declaration of Helsinki.

Of all eyes with retinal detachment, we collected the following data: age, sex, the number of quadrants the retina 
was detached, whether or not the primary retinal detachment was complicated by proliferative vitreoretinopathy (PVR), and whether or not a redetachment occurred. When present, PVR was graded according to the classification system of the Retina Society Terminology Committee. By carefully interrogating the patient, the precise length of time between occurrence of retinal detachment and sampling was established. Some patients were, however, not aware that they had a visual field defect or scotoma caused by the retinal detachment, and were not able to give the exact date. Therefore, the duration of the detachment before surgery was categorised into the following five categories: less than 3 days, 4-7 days, 8-14 days, 2-4 weeks, and more than 1 month, as described earlier. ${ }^{12}$

\section{Sample collection}

Undiluted vitrectomy samples (approximately $0.5 \mathrm{ml}$ ) were obtained by a conventional three port closed vitrectomy technique by manual suction at the beginning of the vitrectomy, before opening the infusion line of balanced salt solution (BSS, Alcon Laboratories, TX, USA). Undiluted subretinal fluid samples were obtained after a scleral puncture, with a 25 gauge bent needle. Samples, which were contaminated with macroscopic haemorrhage, were discarded. The specimens were collected in sterile Eppendorf tubes (Eppendorf, Hamburg, Germany), divided in $100 \mu \mathrm{l}$ aliquots and rapidly frozen and stored at $-80^{\circ} \mathrm{C}$. Sample volumes ranged from $100 \mu \mathrm{l}$ to $1 \mathrm{ml}$.

\section{cGMP radioimmunoassay (RIA)}

cGMP levels in ocular fluid samples were measured as described earlier ${ }^{13}$ using the radio immunoassay procedure according to Brooker $e t$ a ${ }^{14}$ with a polyclonal cGMP antibody made in our laboratory and developed according to Steiner $e t$ al. ${ }^{15}$ Positive controls included buffer containing a range of cGMP (Sigma Chemicals, St Louis, MO, USA), which was used to generate a standard curve. Briefly, samples of patients and controls were diluted 20 times in a $50 \mathrm{mM}$ acetate buffer $(\mathrm{pH}$ 6.5, final volume: $100 \mu \mathrm{l}$ ). Five $\mu \mathrm{l}$ of a mixture of acetic anhydride and tri-ethyleneamine (Merck, Darmstadt, Germany) (1:2) was added and vortexed immediately. Fifty $\mu \mathrm{l}(0.5 \mathrm{kBq})$ of radioactively labelled cGMP (CGMP- ${ }^{125} \mathrm{I}$ ) were added followed by $150 \mu \mathrm{l}$ anti-cGMP antibody (Steiner et al ${ }^{15}$ (dilution 1:30 000) in 0.5\% horse serum (Gibco BRL, Breda, Netherlands). Incubation was overnight at $4^{\circ} \mathrm{C}$. Subsequently, $50 \mu \mathrm{l}$ of undiluted horse serum and $1 \mathrm{ml} 20 \%$ polyethylene glycol (Sigma Chemicals, St Louis, MO, USA) were added to precipitate cGMP containing immune complexes and the reaction mixture was centrifuged at $4^{\circ} \mathrm{C}$ for 20 minutes $(4000 \mathrm{~g})$. The supernatant was removed and the activity of the pellet (containing immunoprecipitated CGMP) was counted in an automatic gamma counter. Each vitreous sample was measured in triplicate. Negative controls included samples to which no cGMP antibody had been added. To determine the amount of cGMP in the samples, the readings were compared to the standard curve generated with the positive controls-that is, the final cGMP concentrations were calculated by comparison against computer fitted curves using known concentrations of cGMP. The amount of cGMP was expressed as nMol (nM) cGMP per litre of undiluted vitreous. The level of sensitivity or limit of detection for this cGMP assay was $0.12 \mathrm{nM}$. The inter-coefficients and intra-coefficients of variation were $7 \%$ and $4 \%$ respectively.

\section{PDE activity}

PDEs are enzymes that are responsible for the degradation of cGMP under physiological conditions. We analysed PDE activity in the ocular fluid samples to determine whether
cGMP was eliminated by high soluble PDE activity. Because there are no data concerning PDE activity in subretinal fluid or vitreous humour, a non-specific, but sensitive PDE assay in order to detect a broad range of soluble PDEs was used in this study. PDE activity was qualitatively measured in subretinal fluid samples and vitreous samples of all patients and controls in triplicate by adding $500 \mathrm{nM}$ cGMP (Sigma chemicals, St Louis, MO, USA) to the vitreous samples and incubating these samples at $37^{\circ} \mathrm{C}$ for 1 hour in Eppendorf tubes (Eppendorf , Hamburg). After 1 hour, incubations were terminated by adding trichloroacetic acid (Sigma chemicals, St Louis, MO, USA), 5\% final concentration. Samples were then extracted five times in succession with $1 \mathrm{ml}$ of water saturated diethylether. In order to remove residual ether, samples were placed for 30 minutes at $50^{\circ} \mathrm{C}$. Subsequently, residual cGMP was quantified using the RIA procedure ${ }^{14}$ described above. As a positive control cerebellar tissue extract (containing $200 \mu \mathrm{g} / \mathrm{ml}$ of tissue protein) was used. This was incubated under similar conditions as the vitreous samples. The level of sensitivity or limit of detection for this assay was $0.12 \mathrm{nM}$. The inter-coefficients and intra-coefficients of variation were $7 \%$ and $4 \%$ respectively.

\section{Total protein}

Total protein concentration of all samples was measured using the method described by Lowry et a ${ }^{16}$ and calculated against a standard curve using bovine serum albumin (BSA) (Sigma chemicals, St Louis, MO, USA).

\section{Statistical analysis}

Results were described with mean (SEM), comparing the patient group with the control group by the $t$ test for independent samples (two tailed). The $t$ test was also used to compare subgroups within the patient groups. The Spearman rank correlation test was used to determine any association between patient age and level of cGMP, and between cGMP levels and total protein concentration, and to determine any association between the duration of the detachment and the cGMP level. Differences were considered significant when the $\mathrm{p}$ value was less than 0.05 (two tailed).

\section{RESULTS}

Vitreous fluid from 50 eyes with retinal detachment was obtained from 50 patients (group I) of which 34 were male and 16 were female. The average age (SEM) in this group was 56.4 (2.2) years (range 14-77 years). Of the 50 eyes, 27 had retinal detachment complicated by PVR grade B and 23 had retinal detachment complicated by grade $\mathrm{CD}$. In the control group of eyes without retinal detachment (group II) eight patients were male and 12 patients were female. The mean age of the control group was 61.0 (2.7) years (range 15-83 years). Subretinal fluid was collected from 70 eyes from 70 patients with a retinal detachment undergoing a scleral buckling procedure (group III). They included 31 (44\%) females and $39(56 \%)$ males. The mean age was $60.0(2.9$ years. Of these 70 eyes, 28 eyes had retinal detachment complicated by PVR grade $B$ to $C_{1}$.

CGMP could be measured in all samples. In vitreous fluid from eyes with retinal detachment (group I) the mean (SEM) level of cGMP was 6.5 (1.7) nM. In the control group without retinal detachment (group II), the mean vitreous level of cGMP was 67.1 (10.0) nM (Fig 1). Thus in patient group I, the mean vitreous level of CGMP was almost 10 times lower than in the control group. This difference was highly significant $(p<0.0001)$. In group III, the mean level of cGMP in subretinal fluid was $2.4(0.2) \mathrm{nM}$. No significant correlation was found between the level of CGMP and age in all three groups. A weak inverse correlation between CGMP and total protein concentration was found in patient group I 


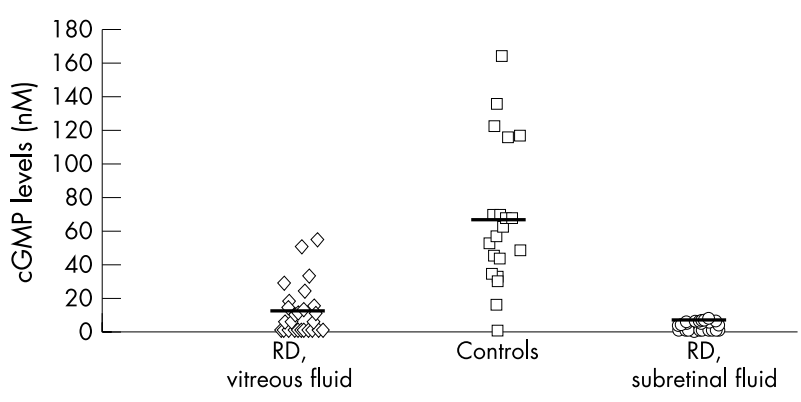

Figure 1 cGMP levels $(\mathrm{nM})$ in vitreous fluid from 50 eyes with retinal detachment (RD) (group I) compared to vitreous cGMP levels in 20 controls without retinal detachment (group II). ( $p<0.0001)$. A third group consisted of subretinal fluid from 70 eyes with retinal detachment (RD) (group III). The horizontal line represents the mean level in each group.

$(r=-0.28, \mathrm{p}<0.05)$. For groups II (controls) and III (subretinal fluid samples) no significant correlation ( $r=0.07, \mathrm{p}=0.753$ and $r=-0.12, \mathrm{p}=0.34$, respectively) was found between cGMP and total protein.

Fourteen of the 70 patients from group III had a recurrent retinal detachment. From five of these 14 eyes with recurrent retinal detachment, undiluted vitreous fluid samples could also be obtained during a subsequent vitrectomy. The mean level of cGMP (2.8 (1.8) nM) was decreased in these samples, and comparable to the cGMP levels found in vitreous fluid from group I.

In patient group I no correlation was found between vitreous CGMP and the duration of the detachment. In patient group III however, we found a significant decrease in the mean subretinal cGMP level when the retinal detachment existed longer $(p<0.0001, r=-0.45$, Spearman rank correlation test); after 1 month mean levels of cGMP were more than three times decreased. In the first 3 days after retinal detachment the mean level of cGMP was 3.6 (0.5) nM, declining after 2 weeks to a mean level of $2.0(0.5) \mathrm{nM}$, and after 1 month to a mean cGMP level of 1.1 (0.2) nM (Fig 2).

In group I no significant difference was found between the vitreous level of cGMP in the nine eyes with less than one quadrant of detachment versus the 41 eyes with two or more quadrants of retina detached. In patient group III, however, the mean level of subretinal cGMP was significantly lower in the 53 eyes with more than one quadrant of retinal

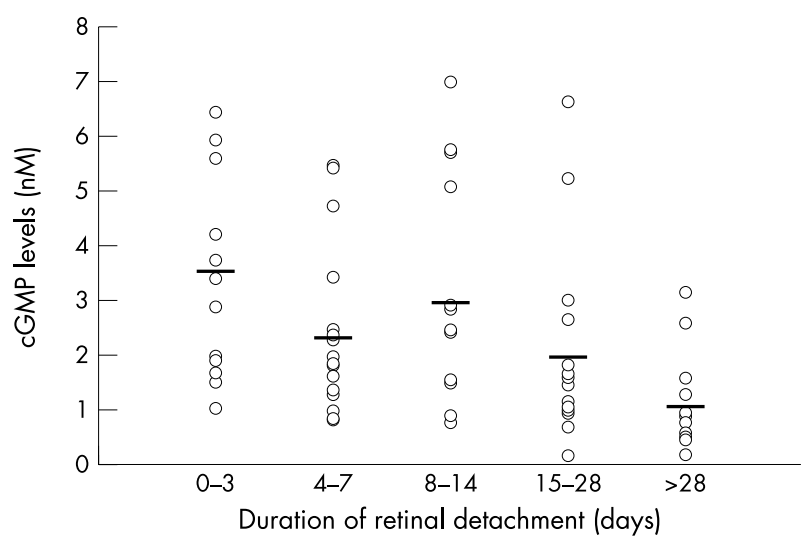

Figure 2 In patient group III, subretinal cGMP levels (nM) correlated moderately with the duration of the retinal detachment. The horizontal line represents the mean level in each group. A decrease in the mean level of cGMP was found if the retinal detachment existed longer. $(p<0.0001 . r=-0.45)$. detachment, mean 2.1 (0.2) nM, compared to the 17 eyes with one or less quadrant of retinal detachment, mean 3.3 (0.5) $\mathrm{nM}(\mathrm{p}=0.03)$.

No PDE activity was detected in any of the samples (patients nor controls) tested, but was readily detected in the positive control under similar conditions.

\section{DISCUSSION}

In this study we found that retinal detachment was associated with a 10-fold decrease in vitreous cGMP concentration. In addition, cGMP levels in subretinal fluid from eyes with retinal detachment were low in all samples. Our study confirms earlier findings in a rabbit model with retinal detachment, ${ }^{10}$ that showed that vitreous cGMP levels were almost $60 \%$ lower than in normal rabbit eyes without detachment. ${ }^{10}$ Because we have not found previous studies on CGMP in normal or pathological human vitreous fluid or subretinal fluid in the literature (computerised search through Medline), we cannot compare our findings with studies in humans.

Although much research in experimental models was done on the role of intracellular cGMP in the retina, little is known about extracellular cGMP. Studies in the kidney however, have indicated that extracellular cGMP has a time dependent and concentration dependent correlation with intracellular CGMP under physiological conditions. ${ }^{17}$

Our study suggests that the low levels of cGMP may be a reflection of degenerating photoreceptors and neuronal retinal cells as a consequence of retinal detachment. Because of hypoxic/ischaemic changes after retinal detachment, ${ }^{45}$ there may be a lower production and release of cGMP by degenerating (photoreceptor) cells with a decreased metabolic cascade. Hypoxia may cause a reduced activity of guanylyl cyclases (GC), which produce cGMP. Thus a decreased amount of CGMP is produced and as a result, less cGMP is released into the extracellular space. The inverse correlation between cGMP and the duration of the detachment in this study supports this.

cGMP is synthesised by guanylyl cyclases (GCs), which are found within photoreceptor cells, in the outer and inner segments. ${ }^{18}$ The soluble isoform of GC (sGC), which is localised in the cytoplasma, is stimulated by nitric oxide (NO). cGMP is also synthesised by (membrane bound) particulate guanylate cyclases (pGC), which are activated by natriuretic peptides. Because of hypoxia within the retina after detachment, the activity of both soluble GC and particulate GC may be reduced, resulting in a lower level of cGMP. In this study we could not discriminate whether CGMP in the vitreous was produced through the NO- sGCcGMP pathway or the natriuretic peptide-pGC-cGMP pathway. Further studies in experimental models using immunocytochemical techniques may give more insight into the role/activity of GCs in the retina of eyes with retinal detachment.

The decreased level of cGMP in eyes with retinal detachment is not the result of increased soluble PDE activity, since no PDE activity could be detected in any of the samples. Because of the retinal hypoxia, it also seems unlikely that an increased activity of intracellular PDEs may have been responsible for a degradation of CGMP within the neuronal cells. Other theories on the undetectable PDE activity in the samples are: (1) levels of PDE activity were below the detection limit of our assay. This seems unlikely since we chose a sensitive PDE assay, (2) PDEs were inactivated or blocked, (3) PDEs are not present in extracellular subretinal fluid or vitreous humour. The difference between not detecting any extracellular PDE activity in the ocular fluid samples, while PDE activity was readily detected in our positive control of cerebellar tissue extract, may be because 
the latter consisted of a tissue homogenate with PDE activity mainly derived from intracellular PDEs. There were no differences in sample collection; the cerebellar tissue extract which was used as a positive control was also first frozen before analysis just like the ocular fluid samples.

We obtained undiluted vitreous samples at the beginning of the vitrectomy, mostly from the anterior part of the vitreous. If one assumes that CGMP in the vitreous body is derived from the retina, there may be a gradient of cGMP, with higher levels at the posterior part of the vitreous and lower levels in the anterior part. This may explain the variation in level of cGMP in the controls, which may be associated with the variation in the depth in the vitreous cavity at which the sample was obtained with the vitrectome.

Most of the subretinal fluid probably results from liquefied vitreous coming across the retinal break. ${ }^{19}$ Aqueous humour may also contribute to the formation of subretinal fluid, because of a flow of aqueous through the retinal hole. ${ }^{20}$ No studies have reported on cGMP levels in normal human aqueous humour. In normal rabbit eyes cGMP levels in aqueous humour were 10 times lower than in vitreous humour. ${ }^{10}$ Despite a small extra amount of cGMP which may have been derived from the aqueous humour however, subretinal cGMP levels in the current study were even lower than vitreous CGMP levels.

Another important aspect of cGMP is its stimulating role in the reabsorption of subretinal fluid by activating the RPE pump. ${ }^{21} 22$ As a result of the decreased levels of cGMP as found in the current study, there may be a slower reabsorption of subretinal fluid, especially in eyes with a longer duration of the detachment. Future intervention studies may therefore aim at modulating subretinal fluid absorption with pharmacological agents that stimulate cGMP, as reported earlier in experimental retinal detachment. ${ }^{212}$

No biochemical markers indicating retinal damage following detachment have been identified yet. Further clinical and experimental studies are needed to investigate whether the observed decreased levels of CGMP in eyes with retinal detachment are a true reflection of a decreased production and release of cGMP from degenerating (photoreceptor) cells in the hypoxic retina after detachment.

\section{ACKNOWLEDGEMENT}

Financial support by the Algemene Nederlandse Vereniging ter voorkoming van Blindheid.

\section{Authors' affiliations}

E C La Heij, H G T Blaauwgeers, A T A Liem, F Hendrikse, University Hospital Maastricht, Department of Ophthalmology, Maastricht, Netherlands
J de Vente, M Markerink, H Steinbusch, Department of Neuroscience A G H Kessels, Department of Clinical Epidemiology and Medical Technology Assessment, University Hospital Maastricht, Netherlands

\section{REFERENCES}

1 Burton TC. Recovery of visual acuity after retinal detachment involving the macula. Trans Am Ophthalmol Soc 1982:80:475-97.

2 Liem ATA, Keunen JEE, van Meel JG, et al. Serial foveal densitometry and visual function after retinal detachment surgery with macular involvement. Ophthalmology 1994;101:1945-52.

3 Sebag J, Sadun AA. Apoptotic photoreceptor cell death after traumatic retinal detachment in humans. Arch Ophthalmol 1996;114:1158-9.

4 Lewis G, Mervin K, Valter K, et al. Limiting the proliferation and reactivity of retinal Muller cells during experimental retinal detachment: the value of oxygen supplementation. Am J Ophthalmol 1999;128:165-72.

5 Mervin K, Valter K, Maslim J, et al. Limiting photoreceptor death and deconstruction during experimental retinal detachment: the value of oxygen supplementation. Am J Ophthalmol 1999;128:155-64.

6 Schraermeyer U, Esser P, Grisanti S, et al. Cytochemical localization of guanylate cyclase in photoreceptor cells of the mouse. Graefes Arch Clin Exp Ophthalmol 1997;235:176-9.

7 Blute TA, Lee HK, Huffmaster T, et al. Localization of natruretic peptides and their activation of particulate guanylate cyclase and nitric oxide synthase in the retina. J Comp Neurol 2000;424:689-700.

8 Newsome DA, Fletcher RT, Chader GJ. Cyclic nucleotides vary by area in the retina and pigmented epithelium of the human and monkey. Invest Ophthalmol Vis Sci 1980;19:864-9.

9 Marmor MF, Kessler R. Sidenafil (Viagra) and ophthalmology. Surv Ophthalmol 1999;44:153-62.

10 Dalma-Weiszhauz J, Blumenkranz M, Hartzer M, et al. Intraocular extracellular cyclic nucleotide concentrations: the influence of vitreous surgery. Graefes Arch Clin Exp Ophthalmol 1993;231:184-6.

11 Retina Society Terminology Committee. The classification of retinal detachment with proliferative vitreoretinopathy. Am J Ophthalmol 1983;90:121-5.

12 Sebag J, Tuyen VV, Faure JP, et al. Retinal S-antigen in human subretinal fluid. Invest Ophthalmol Vis Sci 1987;28:2038-41.

13 Markerink-van Ittersum M, Steinbusch HW, De Vente J. Region-specific developmental patterns of atrial natriuretic factor-and nitric oxide-activated guanylyl cyclases in the postnatal frontal rat brain. Neuroscience 1997;78:571-87.

14 Brooker G, Harper JF, Teraski WL. Moylan retinal detachment. Radioimmunoassay of cyclic AMP and cGMP. In: Brooker G, Green GA, Robinson GA, eds. Retinal detachmentAdvances in cyclic nucleotide research. New York: Raven Press, 1979:1-25.

15 Steiner AJ, Parker CW, Kipnis DM. Radioimmunoassay for cyclic nucleotides. 1.Preparation of antibodies and iodinated cyclic nucleotides. J Biol Chem 1972;247:1106-13.

16 Lowry OH, Rosebrough NJ, Farr AL, et al. Protein measurement with folin reagent. J Biol Chem 1951;193:265-75.

17 Wong KR, Xi MH, Shi LB, et al. Urinary CGMP as biological marker of the renal activity of atrial natriuretic factor. Am J Physiol 1988;255F:1220-4.

18 Blute TA, Lee HK, Huffmaster T, et al. Localization of natruretic peptides and their activation of particulate guanylate cyclase and nitric oxide synthase in the retina. J Comp Neurol 2000;424:689-700.

19 Pederson JE, Toris CB. Experimental retinal detachment. IX Aqueous, vitreous and subretinal protein concentrations. Arch Ophthalmol 1985;103:835-6.

20 Van Heuven WAJ, Kwok-Wai L, Ray S. Source of subretinal fluid on the basis of ascorbate analyses. Arch Ophthalmol 1982;100:976-8.

21 Marmor MF, Negi A. Pharmacologic modification of subretinal fluid absorption in the rabbit eye. Arch Ophthalmol 1986;104:1674-7.

22 Marmor MF. Control of subretinal fluid: experimental and clinical studies. Eye 1990;4:340-4. 